\title{
Biotoxicity Assessment of Incinerator Bottom Ash Leachates Using Daphnia magna
}

\author{
Nan-Min Wu \\ Department of Environmental Engineering and Health, the Yuanpei University of Medical Technology, Taiwan
}

Copyright $\odot 2019$ by authors, all rights reserved. Authors agree that this article remains permanently open access under the terms of the Creative Commons Attribution License 4.0 International License

\begin{abstract}
The Hsin Chu City incinerator generated 35.6 kilotons of incinerator bottom ash (IBA) annually. Most of the IBA were landfilled, while part of them was reused as construction materials for public works. Although the toxicity characteristic leaching procedure (TCLP) of IBA met the Taiwan's current environmental regulations, either landfilled or reused applications were still subject to public concerns. This study evaluated the biotoxicity of IBA leachates using Daphnia magna. The IBA samples were collected from the bottom ash storage pit, following a modified leaching procedure to prepare the leachates. The total elemental analysis of IBA leachates showed that contents of $\mathrm{Cu}$ and $\mathrm{Zn}$ were in the range from $0.35 \%$ and $1.05 \%$, respectively, and were higher than other heavy metals in IBA leachates. In the TCLP testing, all results indicate the metal ions concentrations are well below toxicity standard of the regulation. Daphnia magna was employed to test the static 24-h acute toxicity, and mortality data obtained were further analyzed using the Probit and Spearman-Karber statistical models. Results in the biotoxicity testing indicated that as dilution ratios in $1: 10$, the toxicity unit (TU) values were in the range from 66.7 to 71.4 , and dilution ratios in 1:500, the TU values were in the range from 2.9 to 3.1. The higher the dilution ratios of the leachates, the lower the TU values, indicating that the dilution ratio has a significant effect on the IBA leachates. Experimental results indicated that though the chemical-based TCLP test results were in compliance with regulatory standards, the biological-based toxicity test may reach a certain level of TU value. Accordingly, it is suggested that a biotoxicity program for IBA should be well established in parallel with current TCLP testing to increase the public acceptance for subsequent applications.
\end{abstract} Keywords Incinerator Bottom Ash, Leachate,
Daphnia magna, Toxicity Unit

\section{Introduction}

Incineration is a practical arrangement to treat the municipal solid wastes (MSWs) for more than two decades in Taiwan. It not only readily reduces the volume of MSWs, but also provides substantial energy of electric power supply. However, incineration is ineffectively to treat MSWs that is unsuitable for combustion. As a result, the IBA from MSWs incinerator may contain considerable residues that is probably environmentally risk [1-3]. Partially due to limited land space and up-rising environmental awareness, the IBA has been becoming a heavily loading as secondary pollution derived from incineration.

The IBA is regulated under the Wastes Disposal Act (WDA) in Taiwan. Most of the IBA are landfilled, while part of them are off-site reused as construction materials for public works. Although the toxicity characteristic leaching procedure (TCLP) of IBA met the Taiwan's WDA regulations, the applications are often subject to public concerns.

Currently, there is relatively little information available on toxicity effect on the IBA in Taiwan. It is reported for years that biological activity is directly response to toxic substance, and the use of bioassay has been an alternative and increasing important approach in monitoring the IBA leachates [4-5]. In addition, toxicity identification evaluation is a well-established technique originally developed by the US EPA [6]. Therefore, the objective of this study is to assess biotoxicity of IBA and to identify the possible cause for the observed toxic effect, as it is for subsequent landfill or reuse application.

\section{Materials and Methods}

\subsection{IBA Samples Collection}

The IBA was collected from the MSWs incinerator located in Hsin Chu City, Taiwan. The incinerator received quarter million kilotons of MSWs and generated 
35.6 kilotons of incinerator bottom ash (IBA) annually in average. The incineration procedure producing the IBA is schematically shown in Figure 1 . In order to derive representative samples, the collection procedure was applied according to the method in the National Environmental Analysis Laboratory [7]. Three scheduled samplings were conducted, and all samples of $2 \mathrm{~kg}$ were stored inside well-closed igloo. After samples were all sent back to laboratory refrigerator with temperature-controlled at $7 \pm 2{ }^{\circ} \mathrm{C}$ prior to extraction.

\subsection{Leachate Extraction}

Within 24 hours after arrival to the laboratory, the IBA samples were submitted to the following pre-treatment procedures [8-9]. All lab wares for sample handle and storage were cleaned appropriately to minimize sample contamination.

(1) Well-mixing the IBA samples and divided in five sub-samples;

(2) Drying at $40{ }^{\circ} \mathrm{C}$ for $24 \mathrm{~h}$;

(3) Separating the sample into 4 equal sub-samples;

(4) Taking one sub-sample and sieving to have particle size lower than $5 \mathrm{~mm}$;

(5) Grinding the fraction larger than $5 \mathrm{~mm}$ and repeating step (4) until all IBA size lower than $5 \mathrm{~mm}$;

(6) Weighing $50 \mathrm{~g}$ from step (5) and adding into a $1.5 \mathrm{l}$ Erlenmeyer flask with 11 distill water;

(7) Stirring for $24 \mathrm{~h}$ using a magnetic stirrer;

(8) Filtering the slurry employing a vacuum pump glass fiber filter

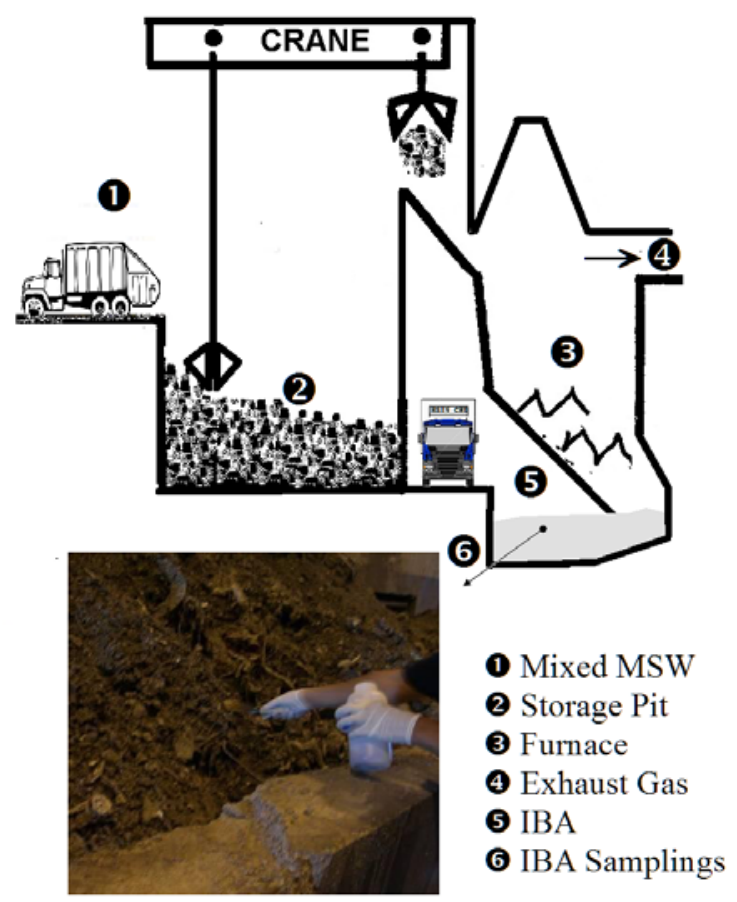

Figure 1. Schematics of the MSWs Incinerator and Samplings Location.

\subsection{TCLP Measurements}

The TCLP measurements were conducted according to the standard method [10]. Leachates obtained from all IBA samples were analyzed to determine whether the IBA is hazardous. Inductively coupled plasma-atomic emission spectrophotometers (Perkin Elma Optima 2100 and Varian Liberty AX) were employed to analyze the concentrations of heavy metal ions in the leachates.

\subsection{Bioassay}

Static 24-h acute toxicity was conducted using Daphnia magna. The stock of Daphnia magna was originally obtained from the Freshwater Aquaculture Research Center, Chu Pei Station (Chu Pei City, Taiwan). Culture conditions were adapted from the Standard Procedure listed in the National Environmental Analysis Laboratory [11]. Culture media was synthetic fresh water $(96.0 \mathrm{mg}$ $\mathrm{NaHCO}_{3}, 60.0 \mathrm{mg} \mathrm{CaSO}+2 \mathrm{H}_{2} \mathrm{O}, 60.0 \mathrm{mg} \mathrm{MgSO}_{4}, 4.0 \mathrm{mg}$ $\mathrm{KCl}$ per liter of double distilled water adjusted to $\mathrm{pH}=7.6 \pm$ 0.2 ) with a hardness in the range of $80 \sim 100 \mathrm{mg} / \mathrm{L}$ as $\mathrm{CaCO}_{3}$. Culture vessels were $3 \mathrm{~L}$ clear glass jars filled with approximately 2 liter of culture media. The neonate daphnia (age $<24 \mathrm{~h}$ ) were obtained from batch cultures maintained in laboratory aquarium at $25 \pm 1{ }^{\circ} \mathrm{C}$ and a 14:10-h light: dark photoperiod with a 15-min transition cycle. Prior to the tests the adult daphnia were separated from the neonates produced that were used in this study. In each test 10 neonate daphnia were exposed to sampled water that was diluted using a 0.5 dilution series $(100,50$, $25,12.5,6.25 \%$ and control). Each test volume was set to $100 \mathrm{ml}$ and was carried out in duplicate. The number of living and dead neonates was noted every 6-h after the initiation of each trial. Neonates daphnia observed as motionless and without a discernable heartbeat were considered to be dead. All $\mathrm{LC}_{50}$ values and their $95 \%$ confidence limits were calculated using the Probit and Spearman-Karber statistical models [8-9]. The Toxicity Unit (TU) is defined as the reciprocal of $\mathrm{LC}_{50}$ multiplying by 100 , or the reciprocal of the dilution ratio for $\mathrm{LC}_{50}$, as shown in equation (1):

$$
\mathrm{TU}=\frac{1}{\mathrm{LC}_{50}} \times 100
$$

\section{Results and Discussions}

\subsection{Total Elemental Analysis of the IBA Leachates}

The composition of IBA leachates may partially reflect the complex of the MSWs fed to the incinerator. Experimental results for the total elemental analysis of IBA leachates are listed in Table 1 . As can be seen, the $\mathrm{pH}$ level is in the range from 11.2 to 11.8 , and can possibly be ascribed to the presence of hydrated lime used as adsorption acidic gas generated in the waste incineration process. Thus, the ratios of $\mathrm{Ca}, \mathrm{Al}$, and $\mathrm{Fe}$ in the IBA are 
higher than other elements. The loss of ignition (L.O.I.) is between $2.47 \% \sim 4.25 \%$, indicates that the IBA leachates contain a certain amount of organic substances. The contents of $\mathrm{Cu}$ and $\mathrm{Zn}$ are in the range from $0.35 \%$ and $1.05 \%$, respectively, and are obviously higher than other heavy metals. Previous studies [13-14] have indicated that the $\mathrm{Cu}$ and $\mathrm{Zn}$ in IBA may mostly exist in carbonate bound form, and are sensitive to environmental conditions, especially $\mathrm{pH}$ values. As the $\mathrm{pH}$ decreased, they can be easily re-released into the environment. Therefore, it is important to evaluate the IBA application facilities and manners so as to avoid potential leaching of biotoxicity.

Table 1. Total Elemental Analysis for the IBA Leachates.

\begin{tabular}{|c|c|c|c|c|}
\hline Items & Unit & IBA 1 & IBA 2 & IBA 3 \\
\hline $\begin{array}{l}\text { Moisture } \\
\text { Content }\end{array}$ & \multirow{2}{*}{$\%$} & 3.23 & 3.25 & 3.55 \\
\hline $\begin{array}{l}\text { Loss of } \\
\text { Ignition }\end{array}$ & & 4.25 & 3.21 & 2.47 \\
\hline $\mathrm{pH}$ Value & - & 11.2 & 11.8 & 11.5 \\
\hline $\mathrm{Al}$ & \multirow{18}{*}{$\mathrm{mg} / \mathrm{kg}$} & 35928 & 30754 & 27453 \\
\hline $\mathrm{Ba}$ & & 2270 & 1897 & 1485 \\
\hline $\mathrm{Ca}$ & & 178443 & 196226 & 210846 \\
\hline Cd & & 0.43 & 0.52 & 0.74 \\
\hline Co & & 56.91 & 110 & 87.41 \\
\hline $\mathrm{Cr}$ & & 257 & 214 & 197 \\
\hline $\mathrm{Cu}$ & & 3579 & 4658 & 4478 \\
\hline $\mathrm{Fe}$ & & 21478 & 25795 & 29872 \\
\hline K & & 15.62 & 10395 & 2084 \\
\hline $\mathrm{Li}$ & & 336 & 442 & 378 \\
\hline $\mathrm{Mn}$ & & 716 & 821 & 1048 \\
\hline $\mathrm{Ni}$ & & 276 & 394 & 457 \\
\hline $\mathrm{Pb}$ & & 950 & 1439 & 1354 \\
\hline $\mathrm{Sb}$ & & 63.83 & 89.14 & 84.17 \\
\hline $\mathrm{Si}$ & & 640 & 1646 & 2142 \\
\hline $\mathrm{Sr}$ & & 341 & 337 & 395 \\
\hline $\mathrm{Ti}$ & & 1104 & 712 & 695 \\
\hline $\mathrm{Zn}$ & & 10637 & 10639 & 10479 \\
\hline
\end{tabular}

\subsection{TCLP of IBA Leachates}

The measured heavy metal ions of the IBA leachates are summarized in Table 2 . Of the nine metals regulated by the WDA, only $\mathrm{Cr}^{6+}$, As and $\mathrm{Hg}$ are not detected above their respective detection limits. As seen from Table 2, metal concentrations of $\mathrm{Cr}$, and $\mathrm{Cu}$ are greater in the TCLP testing, whereas $\mathrm{Ba}$ and Se are the smaller. From Table 2, it is apparent that all the metal ions concentrations are well below toxicity standard of the WDA. Recent studies [12] have found that TCLP does not consistently provide the most conservative estimate of leaching, and its ability to represent worst-case leaching conditions for IBA can be large limited.

\subsection{Biotoxicity Tests of IBA Leachate}

Biotoxicity is a comprehensive evaluation usually used to understand the extent of their biological impact on the environment. By observing the mortality of Daphnia magna of the IBA leachates for 24h, the results of LC 50 can be calculated, so as to obtain the TU values. A series of dilution ratios, e.g. 1:10, 1:20, 1:50, 1:100, 1:200, and $1: 500$, were prepared in conducting the biotoxicity testing. Figure 2 shows the results of TU values with various dilution ratio for the IBA leachates. As can be seen, for the three IBA leachates in same dilution ratio response to a similar range of TU values. As dilution ratios in 1:10, the TU values are in the range from 66.7 to 71.4 , while dilution ratios in 1:500, the TU values are in the range from 2.9 to 3.1. The higher the dilution ratios of the leachates, the lower the TU values. It is clear that the concentration of IBA leachates has a negative effect on the biotoxicity. This higher dilution levels will result in decreasing the quantity of IBA leachates applied in the biotoxicity testing, leading to a lower mortality of Daphnia magna and lower TU values. In addition, the dilution ration in 1:20 can be considered as in the TCLP testing, since the later has a solid: liquid ratio as 1:20 carried in the extraction step. As indicated in previous section, though the TCLPs of all IBA leachates are well below toxicity standard of the WDA, the TU values in this study are in the range from 41.7 to 45.5 . Accordingly, it is suggested that biotoxicity testing should be conducted in parallel with TCLP testing before the IBA to be landfilled or reused as construction material.

Table 2. Summary of Metal Concentration by TCLP for the IBA Leachates

\begin{tabular}{|c|c|c|c|c|c|}
\hline Metals & Unit & Standard & IBA 1 & IBA 2 & IBA 3 \\
\hline As & \multirow{9}{*}{$\mathrm{mg} / \mathrm{l}$} & 5.0 & ND & ND & ND \\
\hline $\mathrm{Ba}$ & & 100 & 0.045 & 0.059 & 0.038 \\
\hline $\mathrm{Cd}$ & & 4.0 & 0.415 & 0.592 & 0.348 \\
\hline $\mathrm{Cr}$ & & 5.0 & 0.556 & 0.305 & 0.424 \\
\hline $\mathrm{Cr}^{6+}$ & & 5.0 & ND & ND & ND \\
\hline $\mathrm{Cu}$ & & 15.0 & 1.547 & 0.535 & 0.821 \\
\hline $\mathrm{Hg}$ & & 0.2 & ND & ND & ND \\
\hline $\mathrm{Pb}$ & & 5.0 & 0.307 & ND & 0.016 \\
\hline Se & & 1.0 & 0.009 & 0.003 & 0.005 \\
\hline
\end{tabular}

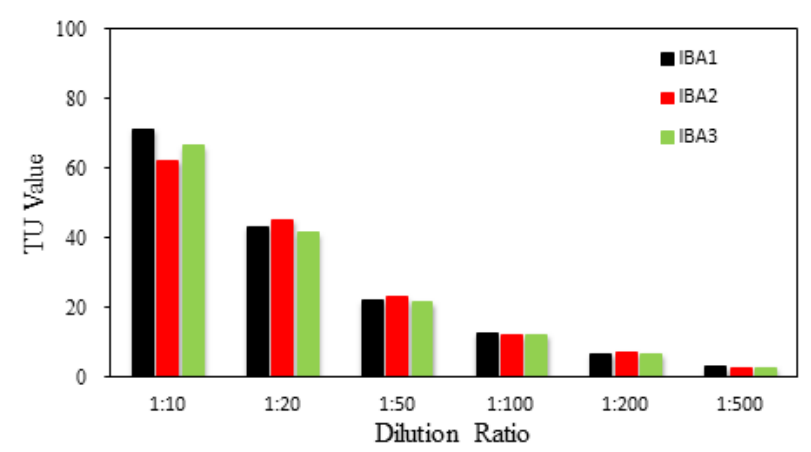

Figure 2. TU Values in Response to Various Dilution Ratio for the IBA Leachates 


\section{Conclusions}

Biotoxicity is a composite index, and can be used to evaluate the IBA leachates. In this study we collected the IBA samples from the incinerator of the Hsin Chu City, Taiwan and conducted biotoxicity test on Daphnia magna. The results showed that the contents of $\mathrm{Cu}$ and $\mathrm{Zn}$ in the IBA are in the range from $0.35 \%$ and $1.05 \%$, respectively, and are obviously higher than other heavy metals. In the TCLP testing, all results indicate the metal ions concentrations are well below toxicity standard of the WDA, Taiwan. While in the biotoxicity testing, as dilution ratios is $1: 10$, the TU values are in the range from 66.7 to 71.4, and dilution ratios in 1:500, the TU values are in the range from 2.9 to 3.1 . The higher the dilution ratios of the leachates, the lower the TU values, indicating that the dilution ratio has a significant effect on the IBA leachates. In comparison with the dilution ration in 1:20 as in the TCLP testing, the TU values are in the range from 41.7 to 45.5. This results indicated that though the chemical-based TCLP test results were in compliance with regulatory standards, the biological-based toxicity test may reach a certain level of TU value. Accordingly, to alleviate the public concerns and to increase the environmental quality, it is suggested that a biotoxicity program for IBA should be conducted in parallel with TCLP testing before considering for subsequent applications, either to be landfilled or to be reused as construction material.

\section{REFERENCES}

[1] S. Patra, S.T. Whaung, and W.L. Kwan, Analysis of heavy metals in Incineration Bottom Ash in Singapore and potential impact of pre-sorting on ash quality, Energy Procedia Vol. 143, (2017). 454-459.

[2] A. M. Joseph, R. Snellings, P. V. den Heede, S. Matthys, and N. De Belie, The Use of Municipal Solid Waste Incineration Ash in Various Building Materials: A Belgian Point of View, Materials. Vol. 11, No.1, (2018). 141-170.

[3] H.W. Luo, Y. Cheng, D.Q. He, and E.H.Yang, Review of leaching behavior of municipal solid waste incineration (MSWI) ash. Vol. 668, No.10 (2019). 90-103.

[4] G. Triffault-Bouchet, B. Clément and G. Blake, Ecotoxicological assessment of pollutant flux released from bottom ash reused in road construction, Aquatic Ecosystem Health \& Management, Vol. 8, No.4, (2005). 405-414.

[5] E. Allegrini, C. Vadenbo, A. Boldrin, and T. F. Astrup., Life cycle assessment of resource recovery from municipal solid waste incineration bottom ash., J. of Environmental Management, Vol. 151, No. 15, (2015), 132-143.

[6] U.S. Environmental Protection Agency, Methods for measuring the acute toxicity of effluents and receiving waters to freshwater and marine organisms, 5th ed., EPA-821-R-02-012 (2002), Washington, DC.
[7] Environmental Analysis Laboratory, Sampling method for the municipal solid wastes incinerator bottom ash, NIEA R119.00C (2004), Taiwan.

[8] C.-C. Lu, M. H. Hsu and Y.-P. Lin., Evaluation of heavy metal leachability of incinerating recycled aggregate and solidification/stabilization products for construction reuse using TCLP, multi-final pH and EDTA-mediated TCLP leaching tests., Journal of Hazardous Materials, Vol. 368, No.15 (2019). 336-344.

[9] H. Kaneko, Evaluation of Municipal Waste Incinerator Fly Ash Toxicity and the Role of Cadmium by Two Aquatic Toxicity Test. Waste Management, Vol. 16, No.5/6, (1996), 555-559.

[10] Environmental Analysis Laboratory, Toxicity Characteristic Leaching Procedure, NIEA R201.15C (2018), Taiwan.

[11] Environmental Analysis Laboratory, Bioassay for acute toxicity using Daphnia magna, NIEA B901.14B (2013), Taiwan.

[12] V. Intrakamhaeng, KA Clavier, J.G. Roessler, and T.G. Townsend. Limitations of the Toxicity Characteristic Leaching Procedure for Providing a Conservative Estimate of Landfilled Municipal Solid Waste Incineration Ash Leaching., Journal of the Air \& Waste Management Association, (2019)

[13] P. Tang, M.V.A.Florea, P. Spiesz, and H.J.H. Brouwers., Application of thermally activated municipal solid waste incineration (MSWI) bottom ash fines as binder substitute, Cement and Concrete Composites, Vol. 70, No. 6, (2016), 194-205.

[14] S. Sorlini, M. C. Collivignarelli, and A. Abbà., Leaching behaviour of municipal solid waste incineration bottom ash: From granular material to monolithic concrete., Waste Management \& Research, Vol. 35, No. 9,(2017), 978-990. 